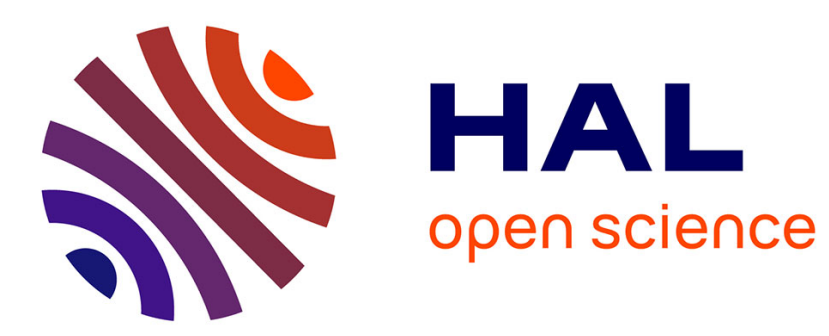

\title{
Dissipation effect on local and global stability of fluid-conveying pipes
}

Olivier Doaré

\section{To cite this version:}

Olivier Doaré. Dissipation effect on local and global stability of fluid-conveying pipes. Journal of Sound and Vibration, 2010, 329 (1), pp.72-83. 10.1016/j.jsv.2009.08.029 . hal-00838862

HAL Id: hal-00838862

https://hal-ensta-paris.archives-ouvertes.fr/hal-00838862

Submitted on 20 Jan 2018

HAL is a multi-disciplinary open access archive for the deposit and dissemination of scientific research documents, whether they are published or not. The documents may come from teaching and research institutions in France or abroad, or from public or private research centers.
L'archive ouverte pluridisciplinaire HAL, est destinée au dépôt et à la diffusion de documents scientifiques de niveau recherche, publiés ou non, émanant des établissements d'enseignement et de recherche français ou étrangers, des laboratoires publics ou privés. 


\title{
Dissipation effect on local and global stability of fluid-conveying pipes
}

\author{
Olivier Doaré \\ ENSTA, Unité de Mécanique, Groupe MS, 91761 Palaiseau Cedex, France
}

\begin{abstract}
In this article, the effect of dissipation on local and global stability of fluid conveying pipes is analyzed. The local approach refers to an infinite medium and uses wave propagation analyses without taking boundary conditions into account. The global approach refers to the same medium, but with finite length and associated with a given set of boundary conditions. The finite length system is generally studied by calculating its eigenmodes and eigenfrequencies. Criteria for local instability are derived in the first part of this paper, and dissipation is found to significantly affect local stability. Moreover, dissipation is found to have a stabilizing or destabilizing effect, depending on the other parameters. Next, numerical computations are presented for finite-length systems and results are analyzed and compared with local stability properties of the corresponding media. When the system is sufficiently long, it is found that critical velocity for global instability tends to a local criterion which can be that of local stability of the damped medium or a local transition criterion of the undamped medium, which is not necessarily the local instability criterion. Finally, a reasoning based on lengthscale ratios is developed. It allows to know which criterion is able to predict the global stability for long systems.
\end{abstract}

\section{Introduction}

As emphasized in a recent review [1], the fluid-conveying pipe is now considered as a model problem for numerous physical systems where the dynamics of a slender structure is coupled to an axial flow. Indeed, it contains most of the physical ingredients found in many other systems, such as shells, plates or cylinders immersed in axial flow, shells conveying fluids or leakage flow problems. The fluid-conveying pipe is known to become unstable at a critical velocity [2], by flutter or buckling, depending on various mechanical parameters and boundary conditions. Two different approaches are commonly used to describe the properties of such systems. The first approach considers the medium to be of infinite length. In this case the waves propagating in the medium are considered through the analysis of the local wave equation. If temporally amplified waves are identified the medium is said to be locally unstable. Depending on the long time impulse response of the locally unstable medium, two types of instabilities may be distinguished: convective or absolute. The concepts of convective and absolute instabilities were first introduced in plasma physics [3], and fruitfully applied to various research fields such as hydrodynamics [4] and fluid-structure interaction [5-7]. The second approach considers the same medium, but of finite length. The modes are studied, through the analysis of the same local wave equation, associated with boundary conditions. If a temporally amplified mode is found, the system is said to be globally unstable.

\footnotetext{
* Tel.: +33169319739; fax: +33169319906

E-mail address: olivier.doare@ensta.fr
} 
The comparison of local and global stability properties has been done on various systems by several authors. One main result that can be drawn from prior studies is that when the length of the system is increased, the critical velocity for global instability tends to a limit that corresponds to a local criterion. However, no unique local criterion can predict the global instability of these long systems. Depending on the medium and the boundary condition characteristics, various authors found that it can be that of absolute instability [8], local instability [9] or that of existence of static or dynamic neutral waves [10,11]. The last criterion may lead to a system that is locally stable but globally unstable. Different theoretical approaches [10,12] explained this unusual result by the existence of an energy gain induced by wave reflexion at boundaries. This phenomenon, called over reflexion, has been observed in other research fields, such as fluid mechanics [13] or astrophysics [14].

In this paper, particular attention will be paid to the influence of dissipation on local and global stability. Regarding wave propagation, some authors have identified that dissipation can have a stabilizing or-more surprisingly-a destabilizing effect. Indeed, it is known after Landahl [15] that neutral waves can be destabilized or stabilized by the addition of damping, depending on the sign of their energy. Regarding fluid-structure interaction systems, it has been found that elastic plates loaded with mean flow also display negative energy waves [5,16,17]. Although the model problem of fluid conveying pipe would probably exhibit similar phenomena, no information is available at present. Conversely, regarding pipes of finite length, dissipation and its effect on global stability has been addressed and destabilization by dissipation has been observed [18,19], but no connection to local wave properties was sought for.

We will focus on the fluid-conveying pipe from now on, with three main objectives: (1) Complete the local and global stability maps of this system. (2) Analyze the particular effect of dissipation on local and global stability. (3) Develop simple criteria for global stability of long pipes based on wave properties analyzes. The motion equations used through the entire paper will be described in Section 2. The third section of the article will be devoted to the wave propagation properties with and without dissipation. The part disregarding dissipation will essentially consist of recalling previous results. Differences between damped and undamped media will be emphasized and analyzed through the calculus of the neutral waves' energy. In Section 4, a numerical Galerkin method will be used to compute the global critical velocities for instability. For long systems, global instability criteria based on lengthscale ratios will be developed. Finally, conclusions will be drawn.

\section{Equations}

The simplest model describing the fluid conveying pipe takes the form of an Euler-Bernoulli beam with an internal plug flow. A schematic view of the system is given in Fig. 1. The linearized equation of motion governing the lateral in-plane deflection $Y(X, T)$ of the pipe is hence [20]

$$
E^{\star} I \frac{\partial^{5} Y}{\partial X^{4} \partial T}+E I \frac{\partial^{4} Y}{\partial X^{4}}+\left(M U^{2}-N\right) \frac{\partial^{2} Y}{\partial X^{2}}+2 M U \frac{\partial^{2} Y}{\partial X \partial T}+c \frac{\partial Y}{\partial T}+(m+M) \frac{\partial^{2} Y}{\partial T^{2}}+S Y=F(X, T),
$$

where $E$ is the Young's modulus, $I$ inertial momentum, $M$ the fluid mass per unit length, $m$ the pipe mass per unit length, $U$ the plug flow velocity, $S$ the elastic foundation modulus, $N$ the external tension applied, $c$ and $E^{\star}$ the viscous and structural dissipations respectively and $F(X, T)$ the external force per unit length. We only consider here the onset of instabilities and nonlinear effects are therefore neglected in the dynamics of the pipe.

In the following, elastic foundation and external tension will be studied separately. Similarly, when damping will be considered, viscous and structural dissipations will be added one at a time. Two different sets of non-dimensional numbers, based on two different sets of characteristic length and time will be used. The first set will be used to study the pipe resting on an elastic foundation, without tension $(N=0)$. Introducing the timescale

$$
\tau=\sqrt{\frac{M+m}{S}}
$$

and the associated characteristic length,

$$
\eta=\left(\frac{E I \tau^{2}}{M+m}\right)^{1 / 4}
$$

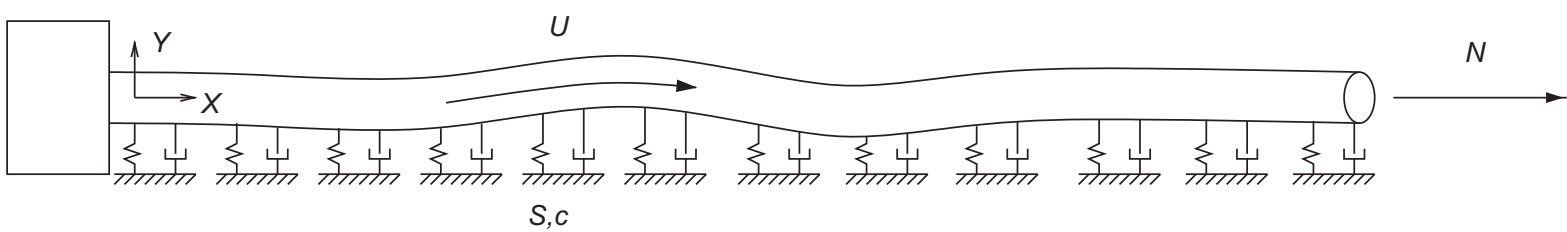

Fig. 1. Schematic view of a cantilevered pipe on an elastic foundation with additional dampers, subjected to an external tension. 
non-dimensional variables and parameters read

$$
x=X / \eta, \quad y=Y / \eta, \quad t=T / \tau, \quad \beta=\frac{M}{M+m}, \quad v=U \sqrt{\beta} \frac{\tau}{\eta}, \quad \alpha=\sqrt{\frac{I}{E(M+m)} \frac{E^{\star}}{\eta^{2}}}, \quad \sigma=\frac{c \eta^{2}}{\sqrt{E I(M+m)}} .
$$

The second set of parameters concern the tensionned pipe. Here, the following characteristic time is used,

$$
\tau=\sqrt{E I(M+m) / N^{2}} .
$$

The lengthscale $\eta$ based on this new characteristic time, is the same as in Eq. (3), and the non-dimensional variables and parameters are the same as in Eq. (4). For both media, the local wave equation reads

$$
\alpha \frac{\partial^{5} y}{\partial x^{4} \partial t}+\frac{\partial^{4} y}{\partial x^{4}}+\left(v^{2}-a\right) \frac{\partial^{2} y}{\partial x^{2}}+2 \sqrt{\beta} v \frac{\partial^{2} y}{\partial x \partial t}+\sigma \frac{\partial y}{\partial t}+\frac{\partial^{2} y}{\partial t^{2}}+b y=f(x, t)
$$

with $(a, b)=(0,1)$ for the pipe on elastic foundation, and $(a, b)=(1,0)$ for the tensionned pipe. For both systems, the length is $L$ and therefore $x \in[0, l]$, with $l=L / \eta$. The particular case of a cantilevered fluid-conveying pipe will be considered through the whole paper. The boundary conditions are consequently those of a clamped-free beam,

$$
y(x=0)=\left.\frac{\partial y}{\partial x}\right|_{x=0}=\left.\frac{\partial^{2} y}{\partial x^{2}}\right|_{x=l}=\left.\frac{\partial^{3} y}{\partial x^{3}}\right|_{x=l}=0 .
$$

The physical meaning of different characteristic times and lengths presented will be discussed in Section 5 .

\section{Local stability}

\subsection{Pipe on elastic foundation}

Looking for solutions in the form of normal modes, $y(x, t)=\mathrm{e}^{\mathrm{i}(k x-\omega t)}$, the local wave equation (6) takes the form of a linear dispersion relation,

$$
D(k, \omega)=(1-\mathrm{i} \alpha \omega) k^{4}-v^{2} k^{2}+2 \sqrt{\beta} v k \omega-\mathrm{i} \sigma \omega-\omega^{2}+1=\phi(k, \omega),
$$

where $\phi(x, t)$ is the Fourier transform in space and time of the forcing function $f(x, t)$. Local stability is ensured if for any real wavenumber $k$, the corresponding frequencies $\omega$, given by Eq. (8) with $\phi=0$, are such that $\operatorname{Im}(\omega) \leq 0$, so that the displacement remains finite in time. When dissipation is absent, the medium is found to be stable if [21]

$$
v<v_{i}=\sqrt{\frac{2}{1-\beta}}
$$

In the local instability domain of the parameters, convective and absolute instabilities may be distinguished. It has been shown [7] that for fluid-structure interaction systems without dissipation, the transition between absolute and convective instabilities arises when the dispersion relation has a triple root. By looking for such triple roots in the instability domain of the parameters, it was found that the medium is absolutely unstable if

$$
v>v_{d}=\left(\frac{12 \beta}{8 / 9-\beta}\right)^{1 / 4}
$$

In the local stability domain of the parameters, one may distinguish between situations where evanescent waves exist at any real frequency to situations where there are some real frequency ranges for which waves are all neutral [10]. These ranges of real frequencies are referred to as neutral, and also arise when the dispersion relation has a triple-root. There are two values of the velocity for which a triple-root exists, $v_{d}$ and $v_{s}=\sqrt{2}$. At the velocity $v=v_{s}$ the neutral range appears at $\omega=0$ and grows symmetrically when $v$ is further increased. It is referred to as static. The frequency range appearing when $v=v_{d}$ does not include $\omega=0$. It is referred to as dynamic. In Fig. 2a, the three velocities $v_{i}, v_{s}$ and $v_{d}$ are plotted as functions of $\beta$. Six domains of wave properties may be then distinguished: absolute instability (AI), when $v>v_{i}$ and $v>v_{d}$, convective instability (CI), when $v_{i}<v<v_{d}$, stability with dynamic range (DN), when $v_{d}<v<v_{i}$, stability with static range (SN), when $v_{s}<v<v_{i}$, stability with both neutral ranges ( $\left.\mathrm{SN}+\mathrm{DN}\right)$, when $v_{d}<v<v_{i}$ and $v_{s}<v$, stability with evanescent waves (E), when $v<v_{s}$.

Now that local properties of waves in the infinite medium without damping have been recalled, the same is done with damping. When the damping is of viscous type, $\alpha=0$ and $\sigma>0$, the local instability criterion has been calculated by Roth [21],

$$
v>\sqrt{2} .
$$


(a)

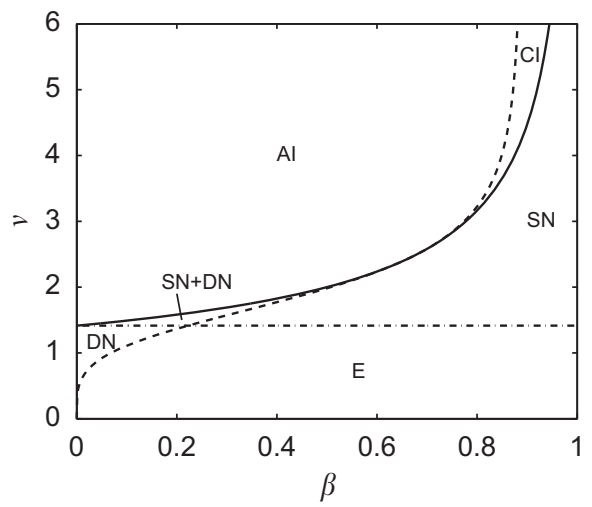

(b)

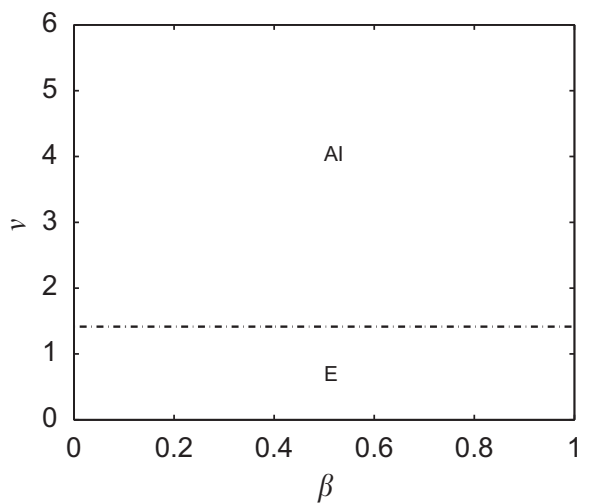

Fig. 2. Pipe on elastic foundation, schematic view of the different domains of wave properties in the $(\beta, v) \mathrm{space} ;(-), v=v_{i} ;(---), v=v_{d} ;(-.-),. v=v_{s} ; \mathrm{AI}$, absolute instability; $\mathrm{CI}$, convective instability; SN, stability with existence of the static range of neutral waves; DN, stability with existence of the dynamic range of neutral waves; E, no neutral wave range exists, there are evanescent waves at any real frequency; (a) without dissipation [10]; (b) with dissipation.

The same calculation is now carried out when structural damping is considered, $\alpha>0$ and $\sigma=0$. Being a second-order polynomial with respect to $\omega$, Eq. (8) has two solutions, $\omega_{+}$and $\omega_{-}$, with the following imaginary parts,

$$
\operatorname{Im}\left(\omega_{ \pm}\right)= \pm \frac{1}{2 \sqrt{2}}\left(\sqrt{\left(-\gamma-A^{2}\right)^{2}+16 \beta k^{2} v^{2} A^{2}}+\gamma+A^{2}\right)^{1 / 2}-\frac{A}{2}
$$

where $\gamma=-(4 \beta-4) k^{2} v^{2}-4 k^{4}-4$ and $A=\alpha k^{4}$. After some trivial algebraic manipulation, it is found that $\operatorname{Im}\left(\omega_{+}\right)>0$ if,

$$
v^{2}>g(k)=\frac{k^{4}+1}{k^{2}} .
$$

The minimum of $g(k)$ is $g(1)=2$. Hence, as soon as $v>\sqrt{2}, \operatorname{Im}[\omega(k)]$ can be positive. The local instability criterion of the pipe with structural damping is thus the same as with viscous damping, given by Eq. (11).

Determination of the nature of the instability, convective or absolute, is less straightforward when dissipation is added. The existence of a third-order root cannot be used anymore to predict convective/absolute instability transition and a numerical analysis of the dispersion relation is necessary in this case. A general procedure is to then carry out a numerical branch analysis of the dispersion relation $[3,4]$. The nature of the instability is determined by the imaginary part of $\omega_{0}$, the frequency at which there is a pinching between $k^{+}$and $k^{-}$branches. Using a systematic branch analysis on the present problem, it is found that $\operatorname{Im}\left(\omega_{0}\right)$ is positive for all values of the velocity parameter $v>\sqrt{2}$, indicating that the instability is absolute.

The domains of local stability and instability in the $(\beta, v)$ plane are plotted in Fig. 2b. Comparison with Fig. 2a leads to the conclusion that dissipation strongly changes the local stability properties of the medium. A remarkable result is that the criterion of stability in the damped case is the same as for the existence of the static range in the undamped case, $v<v_{s}$. Moreover, $v_{i}>v_{s}$, the velocity for instability is consequently lower with dissipation than without dissipation. Analysis of destabilization by dissipation will be more deeply addressed in Section 3.3.

\subsection{Tensionned pipe}

In this section, the same analysis as in the previous section is done on the tensionned pipe, hence the main results will be given with less details. The dispersion relation is here

$$
D(k, \omega)=(1-\mathrm{i} \alpha \omega) k^{4}+\left(1-v^{2}\right) k^{2}+2 \sqrt{\beta} v k \omega-\mathrm{i} \sigma \omega-\omega^{2}=0 .
$$

When dissipation is absent, $\alpha=0$ and $\sigma=0$, following de Langre and Ouvrard [7], the medium is found to be stable if

$$
v<v_{i}=\sqrt{\frac{1}{1-\beta}},
$$

the instability being absolute if

$$
v>v_{d}=\sqrt{\frac{8}{8-9 \beta}}
$$

and convective otherwise. This last critical velocity $v_{d}$ is also a criterion for the existence of a third-order root of the dispersion relation. But here, unlike the previous case of pipe on elastic foundation, $v_{d}$ is always greater that $v_{i}$, and thereby no neutral range appears. However, another criterion for the existence of a third-order root exists, which leads to the 
following criterion for existence of a range of neutral waves,

$$
v>v_{s}=1 \text { and } v<v_{i} .
$$

This range arises at $\omega=0$, hence it is a static range. In Fig. 3a, the three velocities $v_{i}, v_{s}$ and $v_{d}$ are plotted as functions of $\beta$. Four domains of wave properties appear in this figure, absolute instability (AI), when $v>v_{i}$ and $v>v_{d}$, convective instability (CI), when $v_{i}<v<v_{d}$, stability with static range (SN), when $v_{s}<v<v_{i}$, stability with evanescent waves (E), when $v<v_{s}$. This wave properties map closely resembles the corresponding figure for the pipe on elastic foundation. The main difference being that there is no dynamic range of neutral waves in the case with the tensionned pipe.

The stability analysis done in the previous section, involving Eqs. (12) and (13) shows that as soon as $\sigma>0$ or $\alpha>0$, the medium is unstable if

$$
v>1 .
$$

Again, the criterion of instability in the damped case is the same as for the existence of the static range in the undamped case. This criterion is plotted in Fig. 3b. As in the case of the pipe on elastic foundation, damping has a destabilizing effect, here for any value of the mass ratio $\beta$.

\subsection{Wave energy and the effect of dissipation on neutral waves ranges}

The concept of wave energy has been introduced by Landahl [15]. The wave energy of neutral waves is calculated, i.e. $k \in \mathrm{R}, \omega \in \mathrm{R}$ and corresponds to the work done in slowly building up the wave starting from rest at time $t=-\infty$. On the particular problem of flow over campliant surfaces, which exhibits similar equations, such neutral waves and their energy have been extensively studied [5,16]. In particular it is predicted that a negative energy wave (NEW), also referred to as class A disturbance, is destabilized by the addition of damping, while positive energy waves (class $B$ disturbances) are stabilized. Carrying out a study of the Kelvin-Helmholtz instability, Cairns [22] showed that the wave energy $E$ of a neutral wave $y=A \mathrm{e}^{\mathrm{i}(k x-\omega t)}$ is given by

$$
E=e|A|^{2}=-\frac{\omega}{4} \frac{\partial D}{\partial \omega}|A|^{2} .
$$

As this result is independent of the dispersion relation, it can be readily reused in the present case of the fluid-conveying pipe. For both pipes - tensionned and resting on an elastic foundation - the wave energy reads then

$$
e=\frac{\omega}{2}(\omega-\sqrt{\beta} k v) \text {. }
$$

Since $e(\omega=0)=0$, there are NEW in the static range if

$$
\left.\frac{\partial e}{\partial \omega}\right|_{\omega=0}=-\frac{1}{2} \sqrt{\beta} k v<0 .
$$

The parameters $\beta$ and $v$ are both positive, Eq. (21) is thus satisfied when $k>0$. For $\alpha=0, \sigma=0$ and $\omega=0$, the wavenumbers given by the dispersion relation of the pipe on elastic foundation, Eq. (8), are

$$
k= \pm \sqrt{\frac{v^{2} \pm \sqrt{v^{4}-4}}{2}} .
$$

(a)

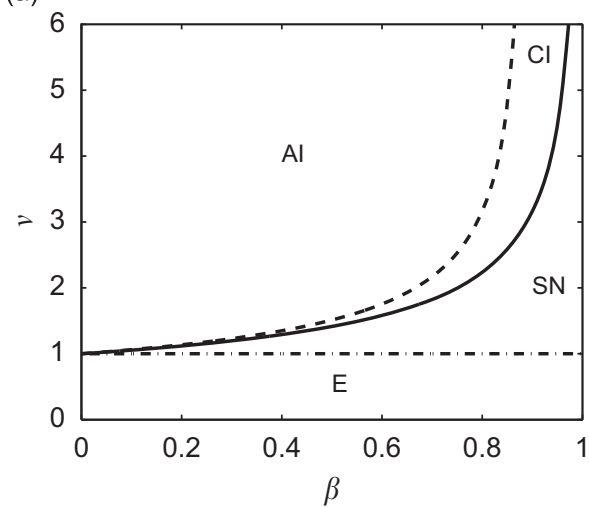

(b)

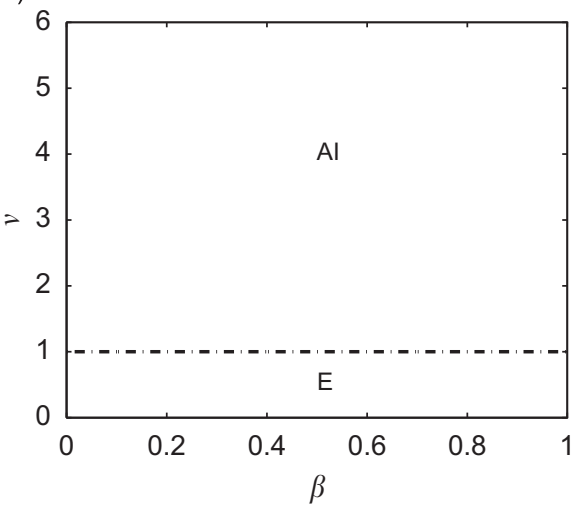

Fig. 3. Tensionned pipe, schematic view of the different domains of wave properties in the $(\beta, v)$ space; $(-), v=v_{i} ;(---), v=v_{d} ;(-\cdot-\cdot), v=v_{s} ;$ AI, absolute instability; CI, convective instability; SN, stability with existence of the static range of neutral waves; E, no neutral waves range exists, there are evanescent waves at any real frequency; (a) without dissipation; (b) with dissipation. 


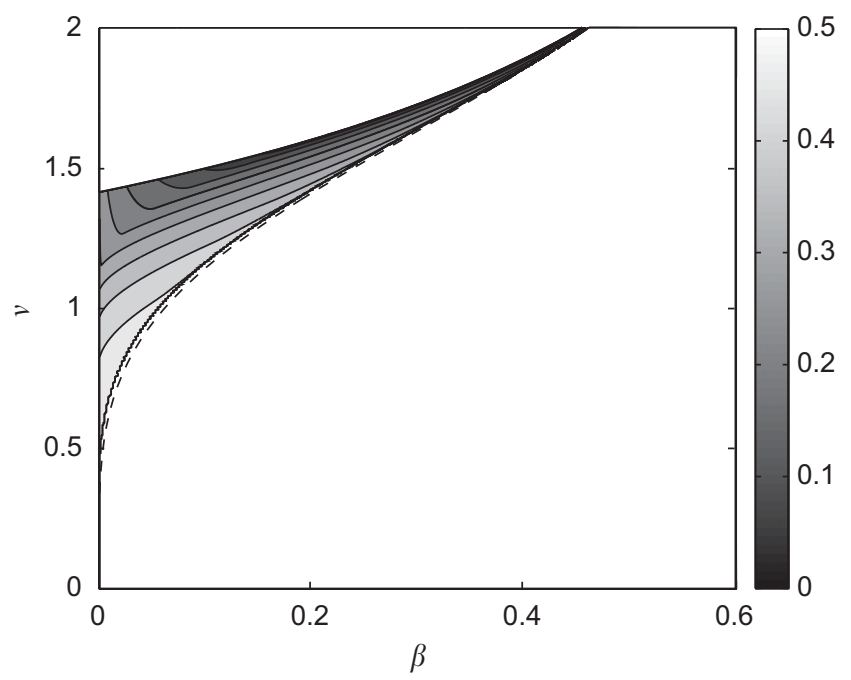

Fig. 4. Pipe on elastic foundation without dissipation, contour plot of the minimum of the wave energy in the domain of the parameters $(\beta, v)$ where the dynamic neutral range exists; (- -), $v_{d}$, critical velocity for existence of the dynamic neutral range; (---), $v_{i}$, critical velocity for local instability.

When $v>\sqrt{2}$, i.e. when the static range exists, two of these wavenumbers are positive. Corresponding waves are hence NEW, which are destabilized by damping.

In the tensionned pipe case, dispersion relation (14) is used with $\alpha=0, \sigma=0$ and $\omega=0$. The wavenumbers are here given by

$$
k= \pm \sqrt{v^{2}-1}
$$

When the static range exists $\left(v^{2}>1\right)$, two of these wavenumbers are positive. Again, corresponding waves are NEW and are destabilized by damping.

As the sign of wave energy in the dynamic range is less straightforward to characterize, a systematic numerical computation has been carried out on the whole domain of existence of the dynamic range. This last domain is defined by Eqs. (9) and (10). In Fig. 4, a contour plot of the minimum value of the wave energy among the four waves in the whole dynamic range is displayed. It appears in this figure that the minimum energy is always positive, so no NEW exists in the dynamic range, which is hence stabilized by the addition of damping.

The wave energy analysis developed in this section shed some light on the effect of dissipation on wave propagation. The first result is that when dissipation is added, some neutral waves in the static range become unstable waves, while the waves in the dynamic range become evanescent waves. Hence, neutral ranges do not exist anymore when dissipation is added. This explains why the local stability maps of Figs. $2 \mathrm{~b}$ and $3 \mathrm{~b}$ are much simpler than those of Figs. 2a and $3 a$. The second result is that the instability due to damping originates from the destabilization of negative energy waves in the static range, which has for consequence that the criterion for local instability with damping is the same as that of existence of the static range when damping is absent, $v>v_{s}$.

\section{Global stability}

Stability of finite length cantilevered pipes is now analyzed. The same media will be considered, with a new physical parameter introduced, the length $L$, and its associated non-dimensional parameter $l=L / \eta$. Boundary conditions are given by Eq. (7). A Galerkin numerical method [23], based on the beam modes, is used to compute the eigenmodes $\phi_{n}$ and eigenfrequencies $\omega_{n}$ of the system, so that the deflection of the pipe, expressed in the base of its eigenmodes, takes the form $y(x, t)=\operatorname{Re}\left[\sum_{n}^{\infty} \phi_{n}(x) \mathrm{e}^{-\mathrm{i} \omega_{n} t}\right]$. The eigenfrequencies $\omega_{n}$ are referred to as global. Instability is predicted when one of them has a positive imaginary part. Up to 150 modes have been used to compute the eigenfrequencies of the longest pipes.

\subsection{Pipe on elastic foundation}

The marginal global stability curves of the pipe on elastic foundation without dissipation are plotted in Fig. 5a for different values of the non-dimensional length $l$. This plot presents similar results as in a previous paper [10], except that higher values of the mass ratio have been explored. As it was highlighted in this previous article, when $l \gg 1$, the global criterion of stability can be well approximated by the local criterion of existence of the dynamic neutral range, $v=v_{d}$. But for $l=16$, one can observe that the global stability curve has an unusual shape when the mass ratio $\beta$ is greater than 0.6 . Some bubbles appear. The transition between global stability and instability is not a single line in the parameters space. 
(a)

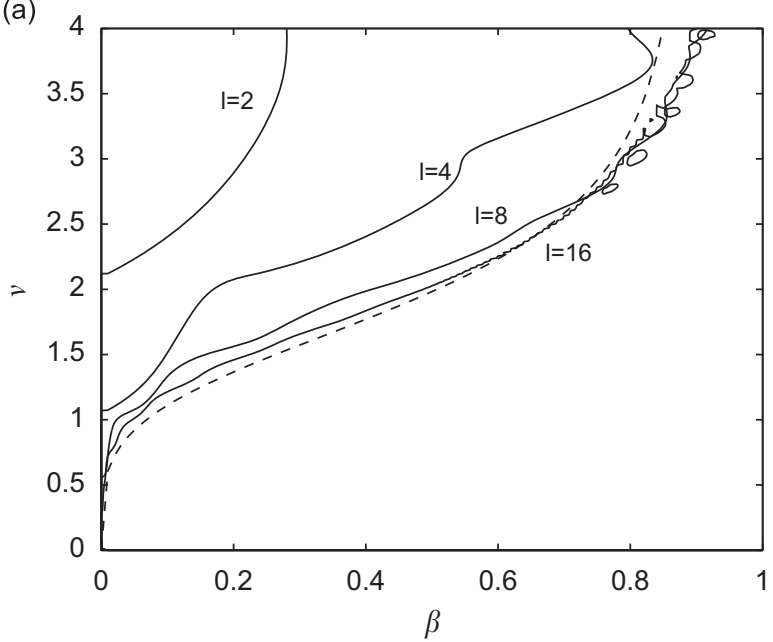

(c)

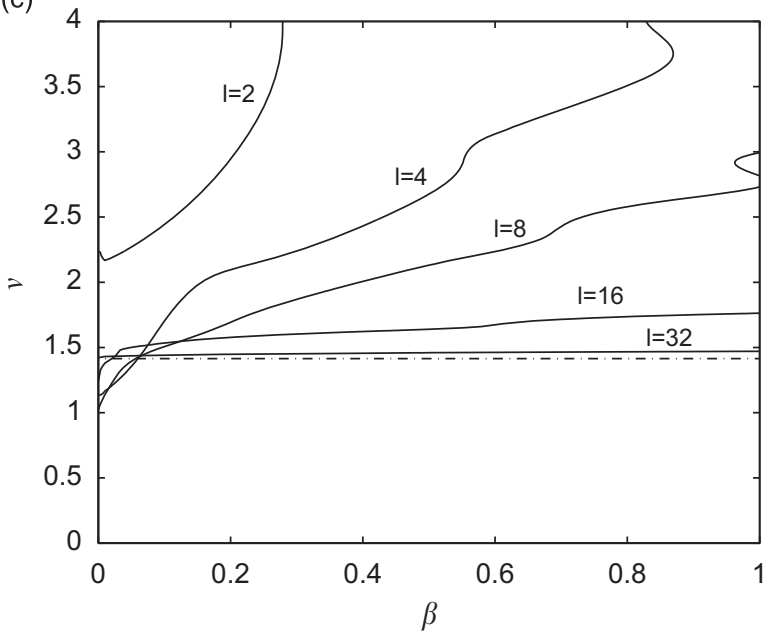

(b)

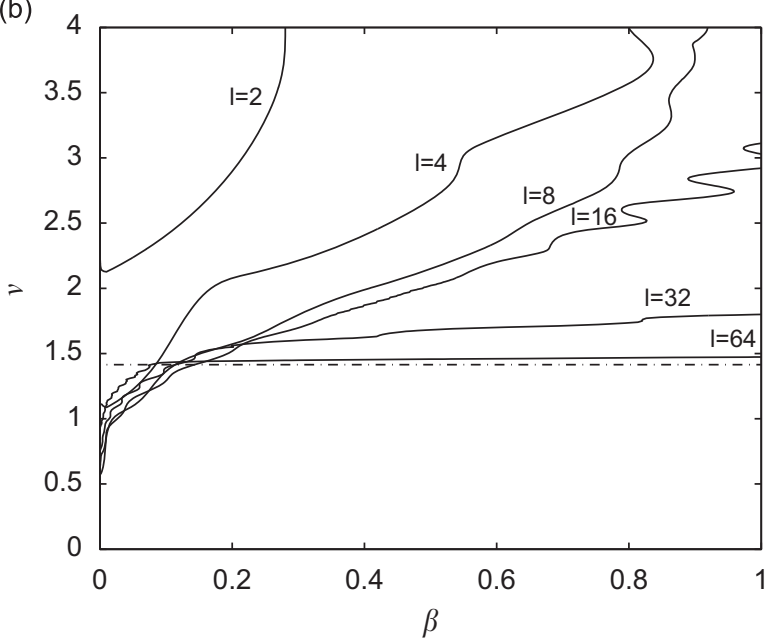

(d)

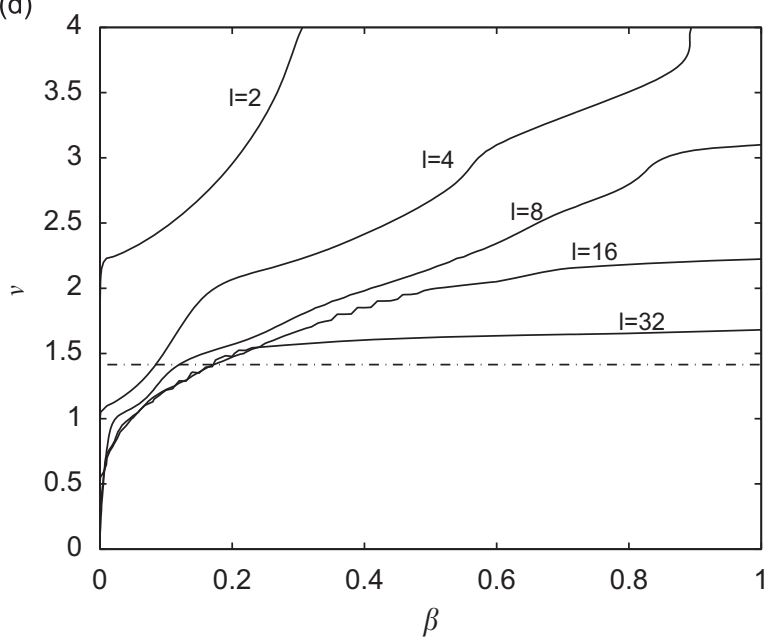

Fig. 5. Fluid-conveying pipe on elastic foundation, global stability curves for different values of the non-dimensional length $l$ and of damping parameters $\sigma$ and $\alpha$. Above these curves, the system is unstable. (---), $v=v_{d}$; (-.-.), $v=v_{s}$; (a) $\sigma=0$ and $\alpha=0$; (b) $\sigma=0.01$ and $\alpha=0$; (c) $\sigma=0.1$ and $\alpha=0$; (d) $\alpha=0.01$ and $\sigma=0$.

Inside the bubbles, one eigenfrequency has a positive imaginary part, indicating that the system is unstable for these values of the parameters. Computations with a very high number of modes have been done to ensure that it is not a numerical artefact. This phenomenon is the same as the one that induces the apparition of loops in global stability curves, first described by Gregory and Païdoussis [23], and is a consequence of successive changes of the number of beam-mode contributions to the eigenmodes of the system.

As dissipation has been found to strongly modify the local properties of stability, it is expected that it will also influence the global stability properties. Local absolute instability will now occur when $v>v_{s}=\sqrt{2}$, due to the destabilization of neutral waves in the static range. Hence it can be predicted that global stability for the long pipe will be determined by this criterion. In Fig. 5b and c, global stability curves are plotted for different lengths and different values of the damping parameters $\sigma$ and $\alpha$. As expected, when the length of the system is increased, the global instability transition approaches the local instability transition $v=v_{s}$.

When $v_{s}>v_{d}$, the addition of dissipation has hence a stabilizing effect, while for $v_{s}<v_{d}$, the addition of dissipation has a destabilizing effect. Transitions between the two regimes, stabilizing or destabilizing effect, occurs when $v_{s}=v_{d}$, at $\beta=\frac{2}{9}$.

\subsection{Tensionned pipe}

Fig. 6a shows the evolution of the marginal stability curve as the length is increased for the tensionned pipe without dissipation. Again, for the long system, the global stability is well predicted by a local criterion. As no dynamic range exists in this medium, the limit for the long system is the local instability transition criterion, $v=v_{i}$. 
(a)

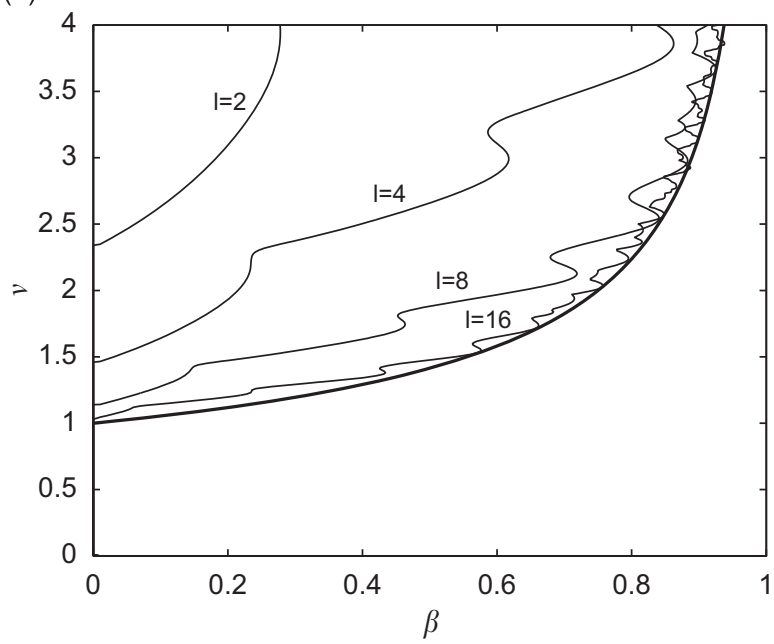

(c)

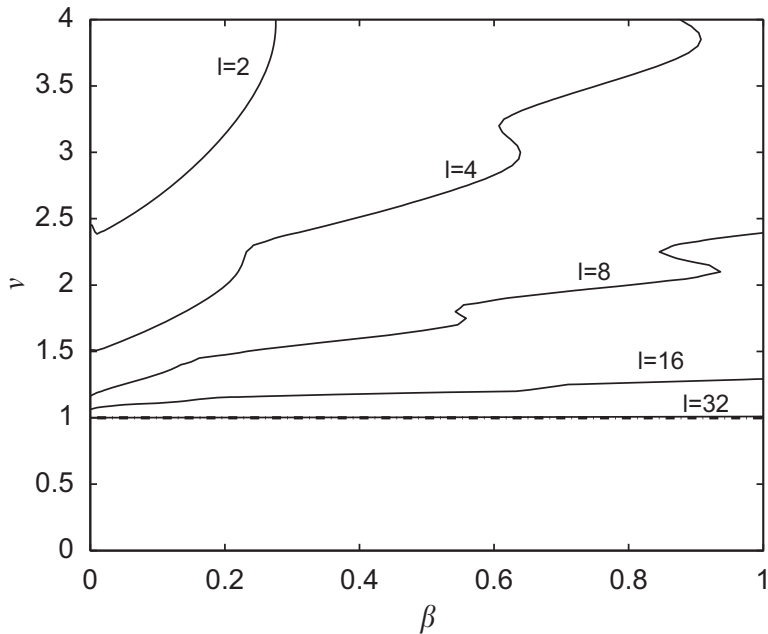

(b)

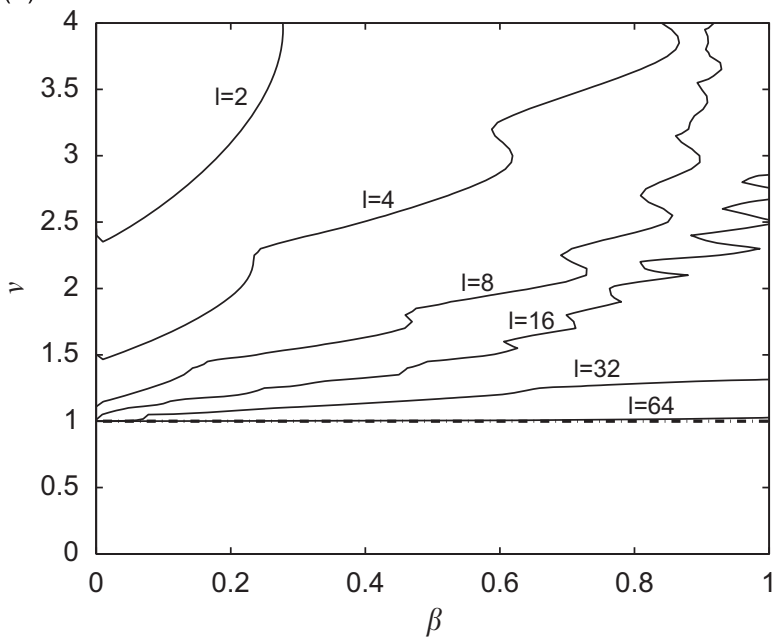

(d)

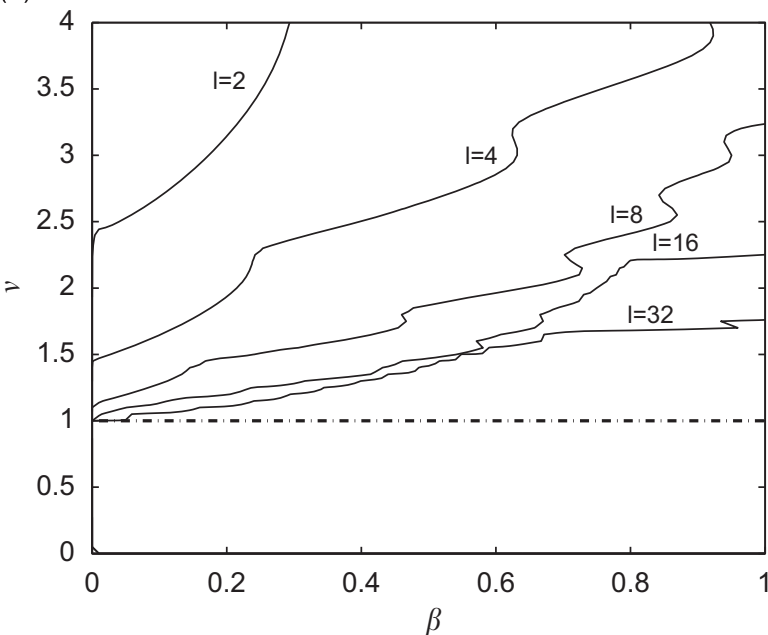

Fig. 6. Tensionned fluid-conveying pipe, global stability curves for different values of the non-dimensional length $l$ and of damping parameters $\sigma$ and $\alpha$. Above these curves the system is unstable. (-), $v=v_{i}$; (-.-.), $v=v_{s}$; (a) $\sigma=0$ and $\alpha=0$; (b) $\sigma=0.01$ and $\alpha=0$; (c) $\sigma=0.1$ and $\alpha=0$; (d) $\alpha=0.01$ and $\sigma=0$.

Like in the previous case of the pipe on elastic foundation, the static neutral range is destabilized by dissipation, so when dissipation is added to the medium, local instability occurs for $v>1$. As it appears in Figs. $6 \mathrm{~b}$ and c, the global marginal stability curve tends to a different limit, which is again the local stability criterion of the damped medium, $v=1$. Here, destabilization by dissipation is observed for any value of the mass ratio $\beta$.

\section{Prediction of global instability using a lengthscale criterion}

It can be observed in Fig. 5b that before merging the local criterion of stability with dissipation, the curve approaches the local criterion without dissipation. This indicates that there exists an intermediate length where the local properties of stability of the undamped medium still dominate the global behavior of the system. To analyze and quantify this phenomenon, the characteristic length and time of each force present in Eq. (1) are now defined. A simple model, based on lengthscale comparisons will be next proposed. We will focus on the pipe on elastic foundation problem. Results obtained here will be directly applicable in the tensionned pipe problem.

The timescale $\tau$, defined in Eq. (2), is the characteristic time associated with the spring foundation and corresponds to the period of oscillation of an unitary-length mass-spring oscillator of mass $M+m$ and rigidity $S$. The lengthscale $\eta$, defined in Eq. (2), is the length appearing in the response of the pipe on elastic foundation without flow, when subjected to the 
transverse unitary forcing $F=\delta(X)[10]$,

$$
Y(X)=\mathrm{e}^{-X / \eta} \sin \left(2 \pi \frac{X}{\eta}\right) .
$$

In the same manner, two other characteristic times $\tau_{\sigma}$ and $\tau_{\alpha}$, associated to the dissipation forces, may be defined. The first mentioned is the relaxation time of waves due to viscous damping

$$
\tau_{\sigma}=\frac{M+m}{c}
$$

while the second is the relaxation time due to structural damping,

$$
\tau_{\alpha}=\frac{M+m}{E^{\star} I} \eta^{4}
$$

Noticeable difference between these two quantities is their dependence on the length $\eta$. Dependence on the wavelength is a well known characteristic of the structural dissipation, and induces a faster attenuation of higher frequency modes in structural vibrations. Conversely, the attenuation by viscous damping is not dependent on the wavelength. Characteristic lengths $\eta_{\sigma}$ and $\eta_{\alpha}$ may now be associated with each dissipation time using the same relation as in Eq. (3),

$$
\eta_{\sigma}=\left(\frac{E I \tau_{\sigma}^{2}}{M+m}\right)^{1 / 4}
$$

and $\tau_{\alpha}$,

$$
\eta_{\alpha}=\left(\frac{E I \tau_{\alpha}^{2}}{M+m}\right)^{1 / 4}
$$

Finally, two non-dimensional lengths may also be defined for the system,

$$
l_{\sigma}=\frac{L}{\eta_{\sigma}}=l \sigma^{1 / 2}, \quad l_{\alpha}=\frac{L}{\eta_{\alpha}}=l \alpha^{1 / 2} .
$$

As pointed out by Carpenter and Garrad [5] in a similar problem of a fluid loaded elastic plate, the merging of the global stability criterion with a local criterion occurs when the most unstable wavelength is small compared to length $L$ of the system. This limit is referred to as the long system limit. In the present problem of fluid-conveying pipe on elastic foundation, the global instability may be driven, in absence of dissipation, by the local instability, for $\beta>\frac{2}{3}$, or over-reflecting boundaries, for $\beta<\frac{2}{3}$. In the first case, using Eq. (8) with $\sigma=0, \alpha=0$ and $\phi=0$, one finds that the first wave which is destabilized as $v$ is increased above the critical value $v_{i}$ has a corresponding wavenumber $k=1$. The corresponding dimensional wavelength is hence $O(\eta)$. In the second case, when global instability is due to wave reflexions at boundary conditions, $\beta<\frac{2}{3}$, the wavelength to consider is that of neutral waves in the dynamic range. Looking for thirdorder roots of the dispersion relation (8) with $\sigma=0, \alpha=0$ and $\phi=0$, one finds that the wavelength of neutral waves at the apparition of the dynamic neutral range is $\lambda=(1 / 4 \pi) \sqrt{24-27 \beta}$, which is also $O(1)$. The corresponding dimensional wavelength is thus $O(\eta)$. Finally, in both cases, $\beta>\frac{2}{3}$ or $<\frac{2}{3}$, the long system limit of the pipe without damping occurs when $L \gg \eta$, that is $l \gg 1$.

In the same manner, the global instability of the system with damping is predicted by a local criterion of the damped medium if $L \gg \eta_{\sigma}$ or $L \gg \eta_{\alpha}$, that is $l_{\sigma} \gg 1$ or $l_{\alpha} \gg 1$, respectively. Hence, when $l \gg 1$ but $l_{\alpha}$ and $l_{\sigma} \ll 1$, it is possible to observe that global instability is predicted by a local criterion of the undamped system, despite the presence of damping. This behavior is expected to arise when $1 \ll l \ll \eta_{\sigma} / \eta$ and $1 \ll l \ll \eta_{\alpha} / \eta$. In order to simplify the discussion, both length ratios, $\eta_{\sigma} / \eta$ and $\eta_{\alpha} / \eta$, will be referred to as $\rho$ in the following. Note that $\rho=\alpha^{-1 / 2}$ or $\sigma^{-1 / 2}$, depending on the type of dissipation in presence.

The critical velocity of global instability is plotted in Fig. 7 as a function of the length parameter $l$ for representative values of the other parameters. In each of these plots, the role of dissipation is emphasized by comparing the critical velocity curves for different values of $\rho$. In all of these plots, the global instability criterion for $l \rightarrow \infty$ is given by the local criterion of the damped medium, except when $\rho=\infty$, i.e. in absence of dissipation. At intermediate lengths, $l \gg 1$ but $l<\rho$, the criterion of instability is that of the undamped system. The results are in agreement with the predictions made above.

In Fig. 8, the length $l_{c}$ at which the critical velocity curve for global instability of the damped system deviates from the curve of the undamped system is plotted as a function of $\rho$. The line $l_{c}=\rho$ is plotted on the same figure for comparison. It appears on this figure that the transition between both local criteria, with and without damping, starts around $l=\rho$. The dispersion between the curves indicates that there exists a slight dependency on $\beta$. Indeed, for values lower than $0.5, l_{c}$ is below the line $l=\rho$, while for higher values, $l_{c}$ is above. This phenomenon remains unpredicted by the simple model presented in this section.

Let us now summarize the methodology developed in this section. One has first to consider a slender structure modelized as an Euler-Bernoulli beam interacting with an axial flow, on which are acting an additional conservative restoring force (elastic foundation or tension) and a dissipative force (structural or viscous). Defining $\eta$ the characteristic length associated with the conservative force, and $\eta_{d}$ the lengthscale associated with the dissipative force, three cases may appear, 
(a)

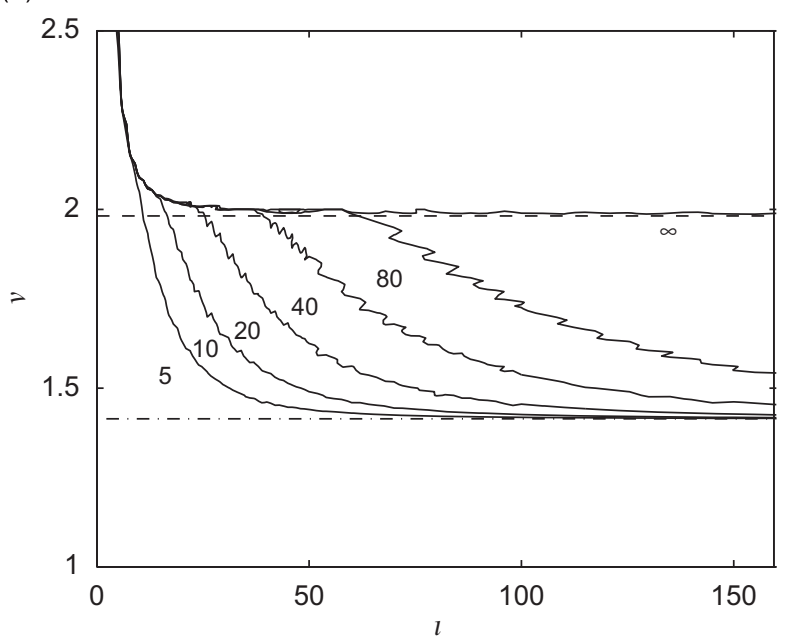

(c)

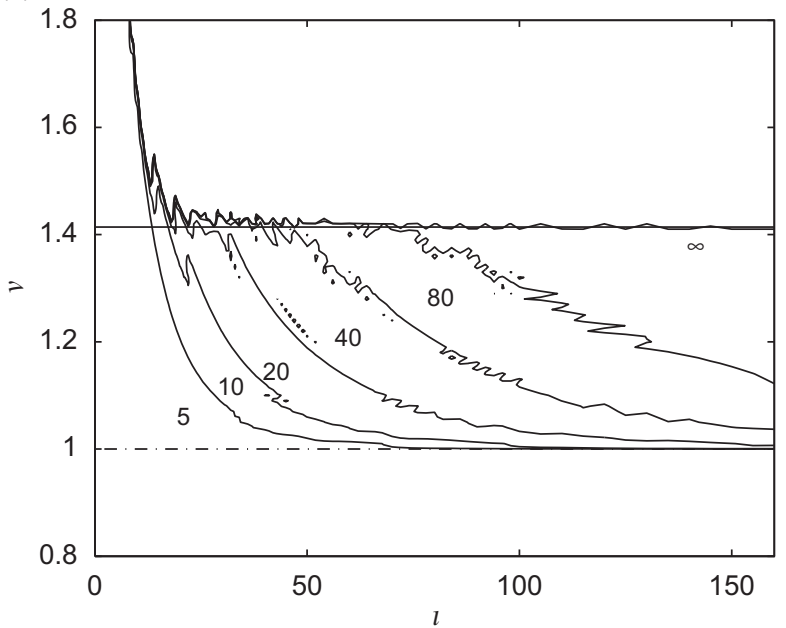

(b)

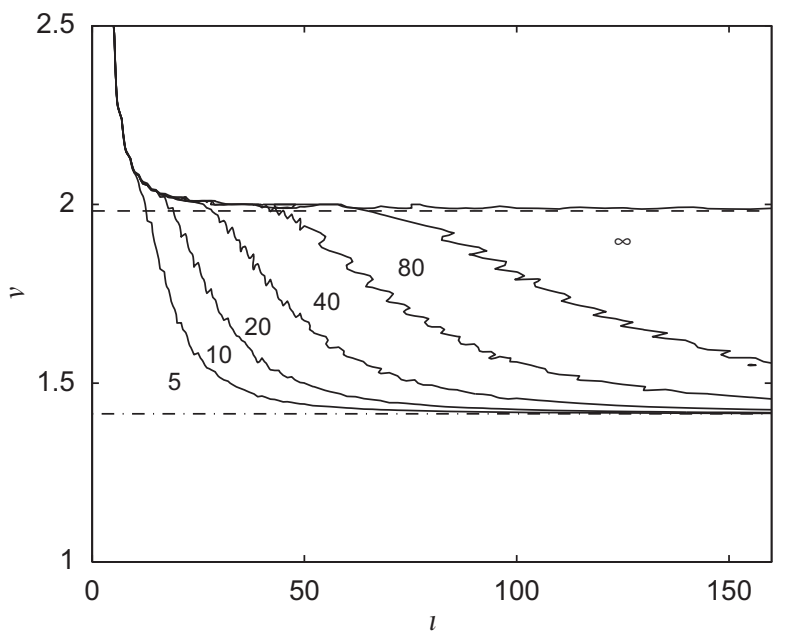

(d)

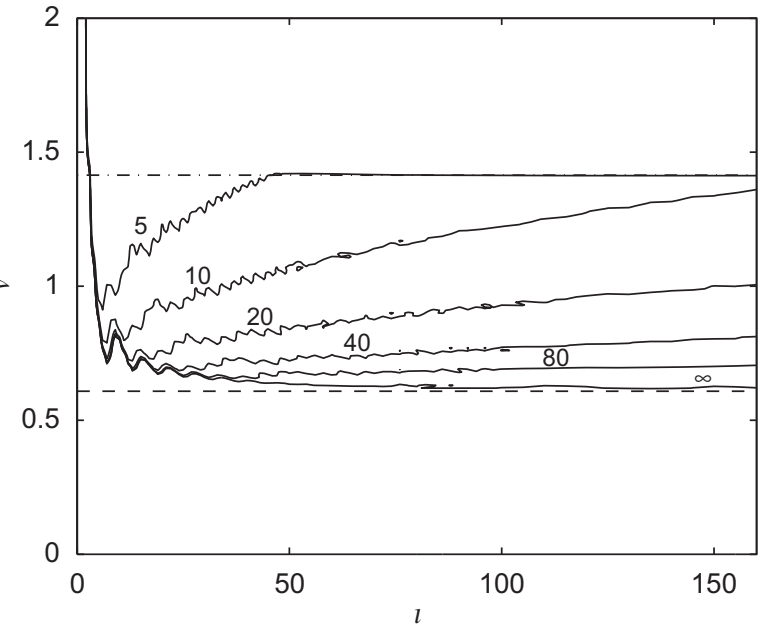

Fig. 7. Non-dimensional critical velocity for global instability as function of the non-dimensional length $l$ for different values of the ratio $\eta_{\sigma} / \eta$ or $\eta_{\alpha} / \eta$; (a) pipe on elastic foundation with viscous damping for $\beta=0.5$; (b) pipe on elastic foundation with structural damping for $\beta=0.5$; (c) tensionned pipe with viscous damping for $\beta=0.5$; (d) pipe on elastic foundation with viscous damping for $\beta=0.01 ;(-), v=v_{i} ;(---), v=v_{d} ;(-\cdot-\cdot), v=v_{s}$.

- If $L \leq \eta$ and $L \leq \eta_{d}$, the system can be considered short, and the global instability can not be predicted by a local criterion.

- If $L \gg \eta$ but $L \leq \eta_{d}$, the local criterion without dissipation predicts global instability:

$$
\text { Instability if } v>v_{i} \text { or } v>v_{d} \text {. }
$$

- If $L \gg \eta_{d}$, the local criterion with dissipation applies:

Instability if $v>v_{s}$.

\section{Conclusion}

Analysis of the effect of damping on the dynamic stability of fluid-conveying pipes has been carried out. Two types of systems have been considered, the fluid-conveying pipe on elastic foundation and the tensionned fluid-conveying pipe. After a complete investigation of the local stability properties in the parameters space, including the recall of previous results, it has been found that the critical velocity for instability of damped media can be much lower than the critical velocity of undamped media. This phenomenon becomes important at high values of the mass ratio. It has then been shown that it is due to the destabilization by dissipation of negative energy waves in a frequency range that was referred to as static in a previous paper [10]. Next, global stability properties have been investigated using numerical Galerkin computations. As the length is increased, the criterion for global instability becomes closely related to the local properties 


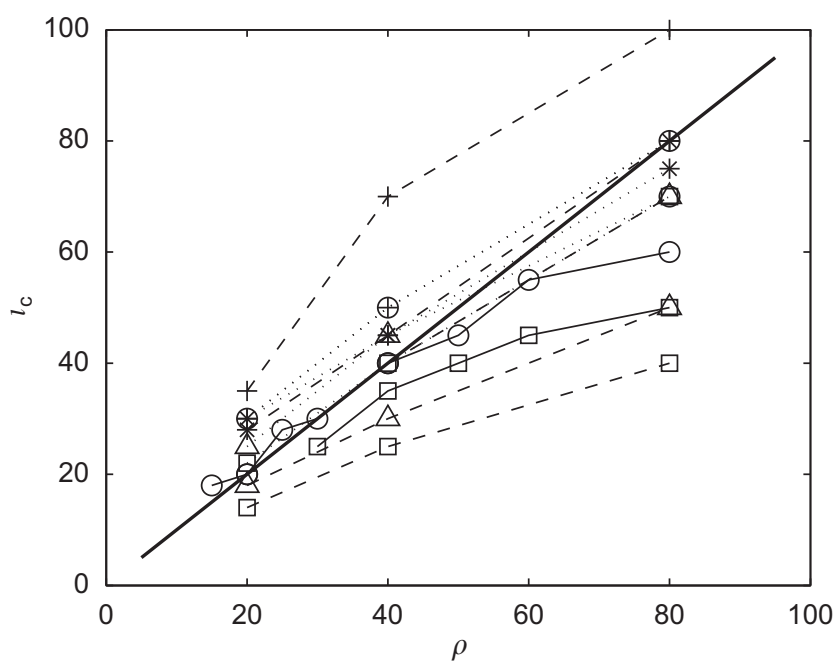

Fig. 8. Non-dimensional length $l_{c}$ at which the critical velocity curve for global instability of the damped system deviates from the curve of the undamped system, as function of $\rho ;(+), \beta=0.3 ;(*), \beta=0.4 ;(), \beta=0.5 ;(\Delta), \beta=0.6 ;(\square), \beta=0.7 ;(---)$, pipe on elastic foundation with viscous damping; (- $)$, pipe on elastic foundation with structural damping; $(\cdots)$, tensionned pipe with viscous damping; bold line, $l_{c}=\rho$.

of the waves in the medium, and it is here found that with damping the critical velocity tends asymptotically to the criterion of local stability. In real situations, damping is always present, giving rise to the question if analyses carried out without damping are at all relevant. But it has been observed in the last section of the present paper that for sufficiently low damping coefficients, the critical velocity for global instability is still predicted by a local criterion of the undamped medium, which is the existence of the dynamic neutral range in the elastic foundation case, and the local instability in the tension case. A reasoning based on lengthscale ratios has been carried out to determine which criterion, with or without damping, predicts global instability.

These results may be applicable in other systems where a slender structure interacts with an axial flow, such as flags, compliant surfaces in presence of a boundary layer, or leakage flow problems. In many of these media, negative energy waves have been identified. Hence, local properties of stability change in the same manner as the present problem of the pipe. It is thus expected that the same will happen to global stability. For these systems, global instability predictions often imply more onerous numerical computations, because of the fact that the characterization of fluid loading due to external flow is a more complicated task than with an internal plug flow-see for instance Ref. [24] for the investigation of a threedimensional potential flow around a flag, or [25], where a new numerical method is proposed to predict instability of a flexible plate in an axial flow. In these problems, local stability analyses associated with lengthscale comparisons could provide simple but sufficient stability criteria.

On the other hand, gravity-related tension has not been considered in the paper. The tension induced by gravity in a hanging slender structure varies linearly, from zero at the downstream end to the weight of the structure at the upstream end. Results presented here would be applicable to the spatially varying media only if the characteristic unstable wavelengths were small compared to the typical length of variation of local properties. It has been shown that it is not the case for the hanging fluid-conveying pipe [26], or the hanging ribbon [27], but some other systems might fall into this category, like slender structures or membranes tensionned by friction [28], or a fluid-conveying pipe where both elastic foundation and gravity-related tension are present.

Destabilization by dissipation has been observed also in the case of finite length fluid-conveying pipe with neither elastic foundation nor tension. But in this case, local instability is observed at any value of the parameters. It is hence not possible to explain a change in the global stability criterion by a change of the local stability criterion. This is currently under investigation.

\section{Acknowledgment}

This work has been funded by the Agence Nationale de la Recherche, under the project "DRAPEAU", number ANR-06JCJC-0087.

\section{References}

[1] M.P. Païdoussis, The canonical problem of the fluid-conveying pipe and radiation of the knowledge gained to other dynamics problems across applied mechanics, Journal of Sound and Vibration 310 (2008) 462-492.

[2] M.P. Païdoussis, Fluid-structure Interactions, Slender Structures and Axial Flow, Vol. 1, Academic Press, New York, 1998. 
[3] A. Bers, Space-time evolution of plasma instabilities-absolute and convective, M.N. Rosenbluth, R.Z. Sagdeev (Eds.), Handbook of Plasmas Physics, Vol. 1, North-Holland, New York, 1983, pp. 451-517.

[4] P. Huerre, M. Rossi, Hydrodynamic instabilities in open flows, Hydrodynamics and Nonlinear Instabilities, Cambridge University Press, Cambridge, 1998.

[5] P.W. Carpenter, A.D. Garrad, The hydrodynamic stability over Kramer-type compliant surfaces. Part 2. Flow-induced surface instabilities, Journal of Fluid Mechanics 170 (1986) 199-232.

[6] A.G. Kulikovskii, I.S. Shikina, On the bending oscillation of a long tube filled with moving fluid, Izvestia Akademii Nauk Arminskoï SSR 41 (1) (1988) 31-39.

[7] E. de Langre, A.E. Ouvrard, Absolute and convective bending instabilities in fluid-conveying pipes, Journal of Fluids and Structures 13 (6) (1999) 663-680.

[8] A.G. Kulikovskii, in: L.D. Landau, E.M. Lifshitz (Eds.), Course of Theoretical Physics, Physical Kinetics, Vol. 10, 1966, p. 281.

[9] O. Doaré, E. de Langre, The role of boundary conditions in the instability of one-dimensional systems, European Journal of Mechanics B-Fluids 25 (6) (2006) 948-959.

[10] O. Doaré, E. de Langre, Local and global instability of fluid-conveying pipes on elastic foundations, Journal of Fluids and Structures 16 (2002) 1-14.

[11] F. Gallaire, J.M. Chomaz, The role of boundary conditions in a simple model of incipient vortex breakdown, Physics of Fluids 16 (2) (2004) $274-286$.

[12] N. Peake, On the unsteady motion of a long fluid-loaded elastic plate with mean flow, Journal of Fluid Mechanics 507 (2004) 335-366.

[13] D.J. Acheson, On over-reflexion, Journal of Fluid Mechanics 77 (3) (1976) 433-472.

[14] J. Andries, M. Goosens, The influence of resonant mhd wave coupling in the boundary layer on the reflection and transmission process, Astronomy and Astrophysics 375 (2001) 1100-1110.

[15] M.T. Landahl, On the stability of laminar a compressible boundary layer over a flexible surface, Journal of Fluid Mechanics 13 (1962) 609-632.

[16] T.B. Benjamin, The threefold classification of unstable disturbances in flexible surfaces bounding inviscid flows, Journal of Fluid Mechanics 16 (1963) $436-450$.

[17] N. Peake, Nonlinear stability of a fluid-loaded elastic plate with mean flow, Journal of Fluid Mechanics 434 (2001) 101-118.

[18] I. Lottati, A. Kornecki, The effect of an elastic foundation and of dissipative forces on the stability of fluid-conveying pipes, Journal of Sound and Vibration 2 (109) (1986) 327-338.

[19] V.M. Vassilev, P.A. Djondjorov, Dynamic stability of viscoelastic pipes on elastic foundations of variable modulus, Journal of Sound and Vibration 297 (1-2) (2006) 414-419.

[20] F.J. Bourrières, Sur un phénomène d'oscillation auto-entretenue en mécanique des fluides réels (On a phenomenon of self-sustained oscillations in the mechanics of real fluids), Publications Scientifiques et Techniques du Ministère de l'Air 147 (1939).

[21] W. Roth, Instabilität durchströmter Rohre (Instability of flow in pipes), Ingenieur Archiv 33 (1964) 236-263.

[22] R.A. Cairns, The role of negative energy waves in some instabilities of parallel flows, Journal of Fluid Mechanics 92 (1979) 1-14.

[23] R.W. Gregory, M.P. Païdoussis, Unstable oscillation of tubular cantilevers conveying fluids, I. Theory, Proceedings of the Royal Society of London, A 293 (1966) 512-527.

[24] C. Eloy, C. Souilliez, L. Schouveiler, Flutter of a rectangular plate, Journal of Fluids and Structures 23 (6) (2007) 904-919.

[25] R.M. Howell, A.D. Lucey, P.W. Carpenter, M.W. Pitman, Interaction between a cantilevered-free flexible plate and ideal flow, Journal of Fluids and Structures 25 (3) (2009) 544-566.

[26] O. Doaré, E. de Langre, The flow-induced instability of long hanging pipes, European Journal of Mechanics A-Solids 21 (2002) 857-867.

[27] C. Lemaitre, P. Hémon, E. de Langre, Instability of a long ribbon hanging in axial air flow, Journal of Fluids and Structures 7 (2005) 913-925.

[28] M.P. Païdoussis, Fluid-structure Interactions, Slender Structures and Axial Flow, Vol. 2, Academic Press, New York, 2004. 